\title{
Intensity Position Modulation for Free-Space Laser Communication System
}

\author{
F. Faghihi and A. Jangjoo \\ Department of Nuclear Engineering, School of Engineering, Shiraz University 71345, Shiraz, Iran
}

\begin{abstract}
In this study a novel analog modulation-demodulation technique for free-space laser communication system called Intensity Position Modulation (IPM) is carried out. According to TEM $_{00}$ mode of a laser beam and by linear fitting on the Gaussian function as an approximation, the variation of the linear part on the reverse biased $p n$ photodiode produced various currents. Our simulated results show fine response bandwidth and fidelity of the analog communication system, is made upon the present method, is satisfactory. According to the present method, we are able to transmit the audio signals up to $1 \mathrm{Km}$.
\end{abstract}

Key words: $\mathrm{TEM}_{00}$ mode, modulation, laser communication, gaussian profile, analog communication, intensity

\section{INTRODUCATION}

Free Space Optical Communication (FSOC) is rapidly evolving area in communication that shows some advantages versus the electrical as security, no requirement for license and low power consumption that recent advances in this field implies the benefits of the method ${ }^{[1]}$.

Different modulation formats such as Amplitude Modulation (AM), Frequency Modulation (FM), Phase Modulation (PM), are used in both the analog and digital systems. In all of them the main properties of the beam such as frequency, amplitude, or intensity changes proportional to the input signals. The required bandwidth depends on the type of the modulation and information signal. So, various modulators are developed and the rate of modulation is restricted by modulator response and also operation ${ }^{[2,3]}$. This research describes Opto-mechanical modulationdemodulation technique for FSOC system that has many differences from the others. In this procedure during the modulation basic properties of the beam remain constant and only its position is changed due to the input information. We will see that the modulated carrier contains no frequency content or in the other words no bandwidth is required to establish communication. As a first experiment, this technique is used here for audio signals of the $50 \mathrm{~Hz}-4 \mathrm{KHz}$ Speech and with respect to the information demodulated at the receiver, operates in a good manner with low distortion.

Linear approximation of the Gaussian function: Principle of the method is based on the linearity of intensity distribution at mode in one - half part of the beam. According to the Gaussian distribution function we can write ${ }^{[4,5]}$ :

$$
\mathrm{I}=\mathrm{I}_{0} \exp \left(\frac{\mathrm{x}^{2}+\mathrm{y}^{2}}{\mathrm{w}^{2}}\right)
$$

In the present research, we assumed a linear fitting to the point of the beam profile that intensity falls $1 / \mathrm{e}^{2}$ on its maximum peak value towards the beam center vicinity, which is shown in Fig. 1. According to the Fig. 1 ; point $\mathrm{A}$ is found by the maximum errors occurred in the linear fitting and point B corresponds to the spot size (w).

\section{MODULATION}

For modulating process of a typical laser beam (e.g. Helium-Neon, or Diode), we used a thin coated mirror which is attached to the spider of a loudspeaker. Signals enter to the coil of the loudspeaker and by interacting the electric flow with magnetic field a force on the mirror is derived. The Modulator setup is shown in Fig. 2. The equation of motion is written as follows ${ }^{[6]}$ :

$$
m \frac{d^{2} x}{d t^{2}}+R_{m} \frac{d x}{d t}+s x=F_{0} \exp (j \omega t)
$$

or:

$$
x(t)=\frac{F_{0}}{\left(s-m \omega^{2}\right)+j \omega R_{m}} \exp (j \omega t)
$$

Where:

$\mathrm{s}=$ Stiffness

$\mathrm{m}=$ Total mass of the vibrator

$\mathrm{R}_{\mathrm{m}}=$ Mechanical resistance

$\mathrm{F} 0=$ Driving force

According to the above equation we can modify displacement of the beam with the angle between the

Corresponding Author: F. Faghihi, Department of Nuclear Engineering, School of Engineering, Shiraz University 71345, Shiraz, Iran , Tel: +987116287500, Fax: +987116287294 
beam and mirror. So, by substituting of Eq. 3 into Eq. 1 we found:

$$
I=I_{0} \exp \left\{-2\left[\frac{(x+x(t))^{2}}{w_{x}^{2}(z)}+\frac{y^{2}}{w_{y}^{2}(z)}\right]\right\}
$$

Displacement of the mirror is assumed parallel to the loudspeaker axis. Thus, great care must be taken into account in the magnet and cone suspender constructions to prevent distortion. In our approach, only the x component of the Gaussian profile varies in the modulation. The dependency of time on beam displacement is described by Eq. 4 .

\section{DETECTION AND DEMODULATION}

A $p n$ photodiode has been used for detection processes. Its dimensions should be smaller than that of the linear part, which is described earlier (Fig. 1). Effective boundaries of the detector are marked by a, b and c (c.f. Fig. 3). It should keep at the center of the linear path to sense the fluctuation of intensity well. The photodiode is sensitive to the average intensity of the beam, thus the total intensity in the steady state will be:

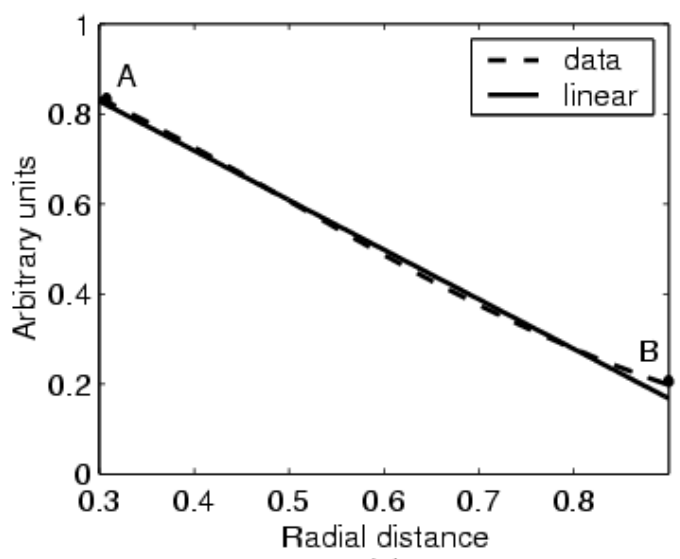

(a)

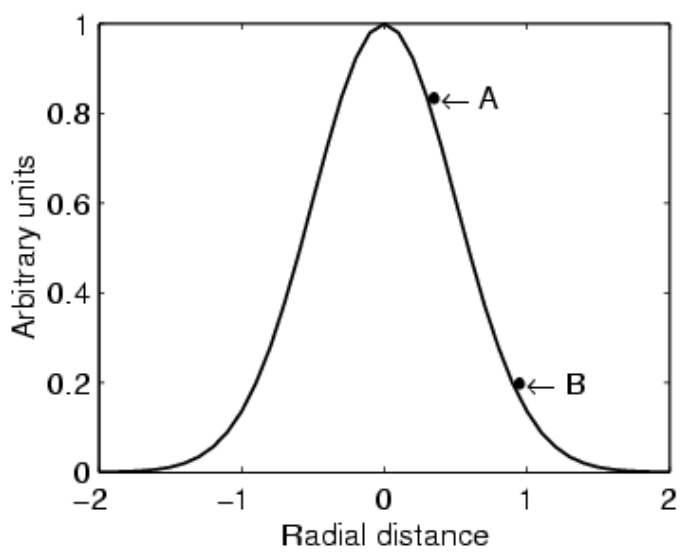

(b)

Fig. 1:Linear fitting on the Gaussian function for beam of $1 \mathrm{~cm}$ spot size (w)
$I_{a v}=I_{0} \int_{a}^{c} \int_{a}^{b} \exp \left\{-2\left[\frac{\left(x^{2}+y^{2}\right)}{w^{2}(z)}\right] d x d y\right.$

and by adding a signal:

$I_{a v}=I_{0} \int_{a}^{c} \int_{a \pm x(t)}^{b \pm x(t)} \exp \left\{-2\left[\frac{\left(x^{2}+y^{2}\right)}{w^{2}(z)}\right] d x d y\right.$

The current - voltage (I - V) characteristic of a pn photodiode follows this equation ${ }^{[7,8]}$ :

$$
\mathrm{I}=\mathrm{I}_{\mathrm{sat}} \exp \frac{\mathrm{qv}}{\mathrm{kT}}-\mathrm{I}_{\mathrm{ph}}
$$

Where:

$$
\begin{array}{ll}
\mathrm{q} & =\text { Electron charge } \\
\mathrm{V} & =\text { Applied voltage } \\
\mathrm{K} & =\text { Boltzmann constant } \\
\mathrm{T} & =\text { Temperature (Kelvin) }
\end{array}
$$

$I_{p h}$ and $I_{\text {sat }}$ are photo induced and saturation currents, respectively. The relation between $\mathrm{I}_{\mathrm{ph}}$ and intensity is ${ }^{[7,8]}$.

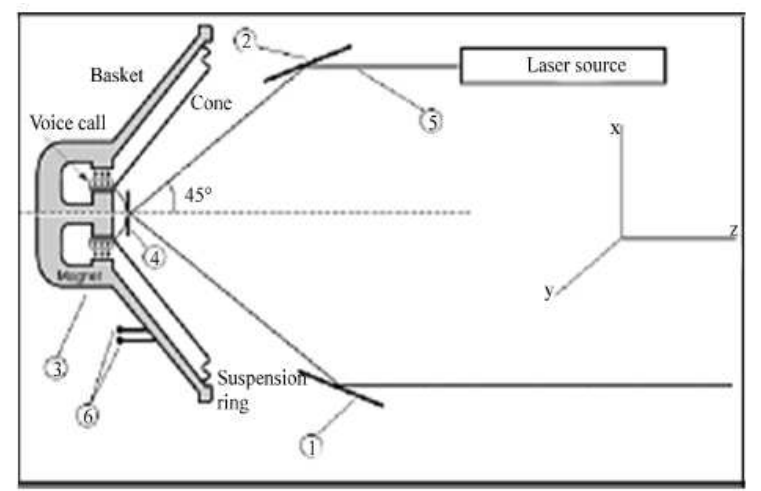

Fig. 2: Modulator components are 1,2: guide mirrors, 3: loudspeaker, 4: vibrating mirror, 5: laser beam at TEM $_{00}$ mode, 6: loudspeaker input connectors. The optimum angle between the beam and vibrating mirror is $45^{\circ}$
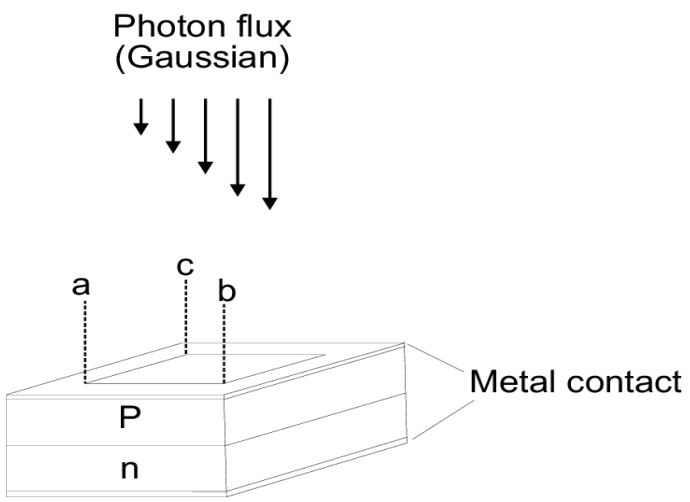

Fig. 3: The scheme of a typical pn photodiode is shown 
$\mathrm{I}_{\mathrm{ph}}=\frac{\alpha \eta \mathrm{A}^{2} \mathrm{Iq} \lambda}{\mathrm{hc}}$

By inserting Eq.6 into Eq.8 we found:

$I_{p h}=\frac{\alpha \eta A^{2} I q \lambda}{h c}\left[I_{0} \int_{a}^{c} \int_{a \pm x(t)}^{b \pm x(t)} \exp \left\{-2\left[\frac{\left(x^{2}+y^{2}\right)}{w^{2}(z)}\right]\right]\right.$

and finally we obtained a relation to time dependent current which is generated by a photodiode:

$I(t)=I_{\text {sat }} \exp \frac{q v}{k T}-\left\{\frac{\alpha \eta A^{2} I q \lambda}{h c} I_{0} \int_{a}^{c} \int_{a \pm x(t)}^{b \pm x(t)} \exp \left[-2\left(\frac{\left(x^{2}+y^{2}\right)}{w^{2}(z)}\right)\right]\right\}$

Where:

$\lambda=$ Wavelength of incident photons

$\mathrm{H}=$ Plank's constant

$\mathrm{c}=$ Velocity of light

$\mathrm{A}=$ Surface area of photodiode

$\alpha=$ Optical loss factor

$\eta=$ Quantum efficiency

Here the photodiode operates in the third quadrant of its (I-V) characteristic and plays as a current controller. Therefore, it should be based with reverse voltage to operate in the linear area, which is shown in Fig. 4.

\section{MODULATION SPECTRUM}

Since in the present method $\mathrm{TEM}_{00}$ mode plays an important role, as discussed earlier, we rewrite the Eq.6 in the form of:

$|E|_{a v}^{2}(t)=\left|E_{0}\right|^{2} \int_{a}^{c b \pm x(t(t)} \int^{b} \exp \left\{-2\left[\frac{\left(x^{2}+y^{2}\right)}{w^{2}(z)}\right]\right\} d x d y$

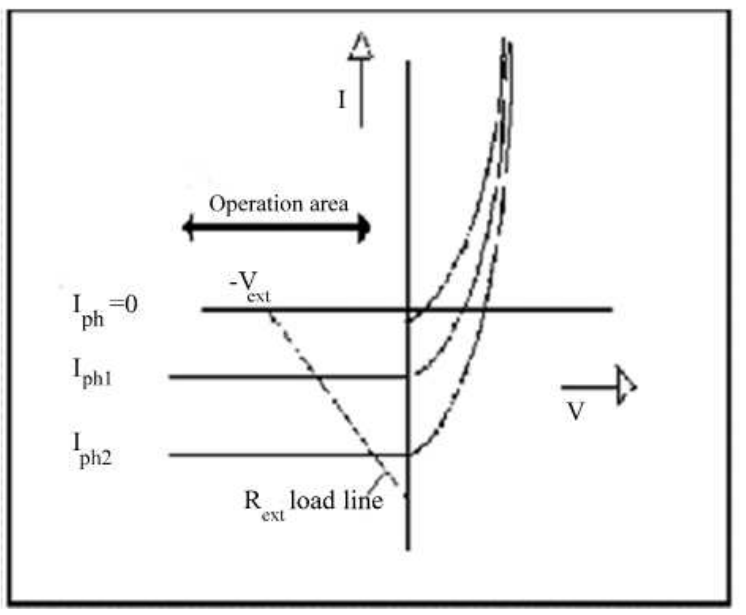

Fig. 4: Current-voltage curve of the $p n$ photodiode
The above equation shows the time evolution of absolute value of the electric field distribution on the $\mathrm{x}$ y surface with no time dependency in the field. In other words, signals do not affect the carrier frequency and we can say that no bandwidth is required for the IPM based communication system. It is mentioned here that the Doppler shift due to the movement of the beam positions causes line broadening but the resulting bandwidth does not contain any information. So, we produced a narrow band beam by filtering and the $p n$ photodiode worked properly for detection by the so called narrow band beam.

\section{MATERIALS AND METHODS}

Typical devices that operate with the cited technique are developed for analog voice communications. A diode laser $(\lambda=630 \mathrm{~nm}$ and $\mathrm{P}<0.5 \mathrm{~mW}$ ), in the $\mathrm{TEM}_{00}$ mode is provided to carry an optical source which is biased with DC power supply. A woofer loudspeaker, which has the best response to frequencies in the range of $50 \mathrm{~Hz}-4 \mathrm{kHz}$, was applied for the modulating. The amplification of the signals performs with analog tone-control preamplifier and power amplifier. To prevent interference with undesired noises, the modulator was shielded by an acoustic insulator. The interference signals with a natural resonance frequency of the loudspeaker are the source of the noises. The tone- control preamplifier can be used to balance the amplitude of the undesired signals. The place of the photodiode as well as the Gaussian shape of the beam and detector operation are shown in Fig. 5.

The amplitude of the signals should be controlled for limiting displacement of the beam. If we have taken into account improper amplitude, it caused beam jumping or amplitude distortion. Signals detected directly by the photodiode and produced current could be amplified by the same amplifier of the modulator. In our experiment, the detector has been located $1 \mathrm{Km}$ far away from the transmitter.

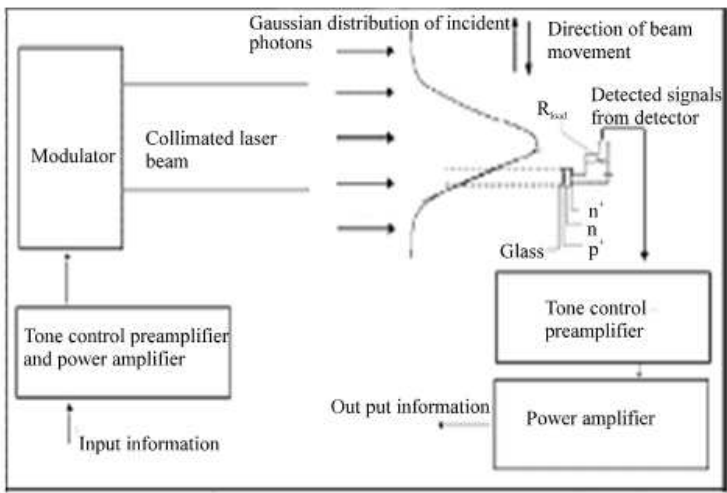

Fig. 5: The operation of a typical voice communication system is illustrated 

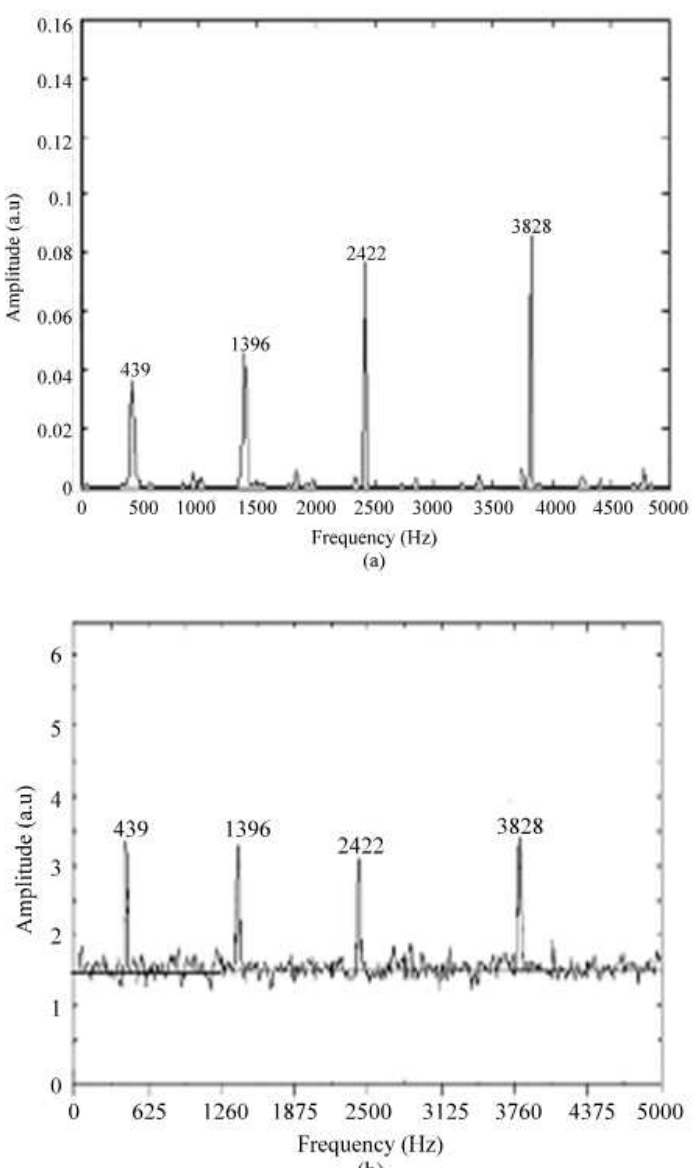

(b)

Fig. 6: FFT spectrum comparison of the four sinusoidal signals after demodulating both experimentally and (b) theoretically

Fast Fourier Transform (FFT) analyzed spectrum of the sample signals after demodulating has been shown in Fig. 6a. The calculated spectrums are found by the Eq. 10 and our results are given by Fig. 6b. FFT spectrum simulation is performed with MATLAB software ${ }^{[9,10]}$ and the experimental data have been obtained by Tektronix (TDS 210) digital oscilloscope.

\section{RESULTS AND CONCLUSION}

We discussed IPM method with the analog format for FSOC system and by the present technique, we modulated the audio signals properly and with low distortion. Experimental results are in good agreement with theoretical predictions coming from the Gaussian profile at the mode. The variety of the atmospheric conditions at far distances will cause a severe distortion. So, we need stability conditions for the intensity profile. Therefore, it seems that the method can be applied for short and medium distances, due to low deformation of the profile ${ }^{[11]}$. Because of the frequency dependent on the term $\left(s-m \omega^{2}\right)+j \omega R_{m}$, the amplitude of demodulated signals were fluctuated by frequency discrimination effect. To avoid any problems, the tone control preamplifier has an important responsibility to balance the gain of the output signals before the modulator. Transmission may be changed by the atmosphere refractive index at hard airflow and causes other cases of perturbation. The suggested solutions are discussed as:

- Double beam configuration

- Using the sub-carrier

If we were using another laser beam, which contains noises only, they could be locked in the amplifier by another detector at the opposite phase to cancel undesired noises. This condition refers to the part I. In the $2^{\text {nd }}$ procedure, a sub-carrier of frequency more than the noise level carries the information.

As a technical feasibility, IPM can be extended to the higher modulation rates by an acoustic-optic modulator for digital communication ${ }^{[12]}$, where it is our goal. Our emphasis here is on a new concept of FSOC and other related fields may be developed by such an acousto-optic modulator.

\section{ACKNOWLEDGMENTS}

This work is financially supported by the Research Council of the Shiraz University under grant No. 83EN-1708-C275. The Corresponding author wishes to acknowledge the Research Council of the Shiraz University.

\section{REFERENCES}

1. Emil Hallstig, 2001. Optical Free Space Communication, Project Work in the Course Optoelectronics. IFM, Linkoping.

2. William K. Pratt, 1969. Laser Communication Systems. John Wiley.

3. Carlson, A.B., P.B. Crilly and J.C. Rutledge, 2002. Communication Systems. 4th Edn., McGraw-Hill.

4. William T. Silfvast, 1996. Laser Fundamentals. Cambridge, Cambridge University Press.

5. Amnon Yariv, 1989. Quantum Electronics. 3rd Edn., John Wiley.

6. Joseph L. Hunter, 1965. Acoustics. Prentice-Hall.

7. Alan Billings, 1993. Optics-Optoelectronics and Photonics. Prentice-Hall.

8. Hans Melchior, Mahlon B. Fisher and Frank R. Arams, 1970. Photodetectors for optical communication systems. Proc. IEEE., 58: 1466.

9. Faghihi, F. and K. Hadad, 2004. Numerical solutions of coupled differential equations using Maple software. Appl. Math. Comp. Method, 155: 563.

10. Faghihi, F., 2005. Numerical minimization procedures of the adiabatic wave function. Appl. Math. Comp. Method, 162: 1013.

11. Morio Toyoshima, et al., 2001. Mutual Alignment Errors Due to the Variation of Wavefront Aberrations in a Free-Space Laser Communication Link. Optics Express, 9: 592.

12. Gordon, E.I., 1966. A review of acousto-optic deflection and modulation devices. Proc. IEEE., 54: 1391 . 